\title{
ON REARRANGEMENTS OF VILENKIN-FOURIER SERIES WHICH PRESERVE ALMOST EVERYWHERE CONVERGENCE
}

\author{
BY
}

\author{
J. A. GOSSELIN AND W. S. YOUNG $\left({ }^{1}\right)$
}

\begin{abstract}
It is known that the partial sums of Vilenkin-Fourier series of $L^{q}$ functions $(q>1)$ converge a.e. In this paper we establish the $L^{2}$ result for a class of rearrangements of the Vilenkin-Fourier series, and the $L^{q}$ result $(1<q<2)$ for a subclass of rearrangements. In the case of the Walsh-Fourier series, these classes include the Kaczmarz rearrangement studied by L. A. Balashov. The $L^{2}$ result for the Kaczmarz rearrangement was first proved by $K$. H. Moon. The techniques of proof involve a modification of the Carleson-Hunt method and estimates on maximal functions of the Hardy-Littlewood type that arise from these rearrangements.
\end{abstract}

1. Introduction. Let $G=\prod_{j=0}^{\infty} Z_{p_{i}}$ denote a countable product of cyclic groups with orders $p_{i}$ and Haar measure $\mu$ normalized so that $\mu(G)=1$. We assume throughout this work that $\sup p_{i}=p<\infty$. For $x=\left(x_{i}\right) \in G$, let $\psi_{j}(x)=$ $\exp \left(2 \pi i x_{j} / p_{j}\right), j=0,1,2, \ldots$ The sequence $\left\{\psi_{j}\right\}$ generates the standard ordering $\left\{x_{n}\right\}$ of the dual group $X$ as follows: Let $m_{0}=1$ and $m_{s}=\prod_{i=0}^{s-1} p_{i}$ for $s=1,2, \ldots$ Then each natural number $n$ has a unique representation of the form $n=\Sigma_{s=0}^{\infty} a_{s} m_{s}$, where $0 \leqslant a_{s}<p_{s}$, and $a_{s}=0$ for sufficiently large $s$. Define $\chi_{0}=1$, and for $n \geqslant 1$ with $n=\Sigma_{s=0}^{\infty} a_{s} m_{s}$, define $\chi_{n}=\Pi_{s=0}^{\infty} \psi_{s}^{a_{s}}$. If $p_{i}=2, i=0,1, \ldots, G$ is the Walsh group $2^{\omega},\left\{\psi_{j}\right\}$ can be identified with the Rademacher functions and $\left\{\chi_{n}\right\}$ the Walsh functions. In general, the system $\left(G,\left\{x_{n}\right\}\right)$ is a special case of the Vilenkin systems studied in [12] and [5]. It follows that if $f \in L^{q}(G), 1<q \leqslant \infty$, then the Fourier series of $f$ with respect to $\left\{x_{n}\right\}$ converges a.e. $(\mu)$ to $f$. In this work we show that a.e. convergence of the Fourier series for $L^{2}(G)$ is preserved by a class of rearrangements of $\left\{\chi_{n}\right\}$ and that a.e. convergence for $L^{q}(G), 1<q<2$, is preserved by a subclass of these rearrangements. In the case of the Walsh system, these classes include the Kaczmarz rearrangement studied by L. A. Balashov [1]. The $L^{2}$ result was first established for the Kaczmarz rearrangement of the Walsh system by K. H. Moon [9]. The $L^{q}$ result was first established for more general rearrangements of the Walsh system in [14]. (See [15].) Finally, we note that the methods used in this

Received by the editors November 26, 1973 and, in revised form, March 27, 1974. AMS(MOS) subject classifications (1970). Primary 43A50; Secondary 42A56.

Key words and phrases. Hardy-Littlewood maximal function, maximal $n$th partial sum operator, conditional expectation, weak type inequalities.

(1) Research supported by NSF Grant GP 32116. 
work are modifications of those of L. Carleson [3] and R. A. Hunt [6], [7].

The authors would like to thank Professor Hunt for his helpful suggestions.

2. Statement of results. We study the a.e. convergence properties of Fourier series with respect to certain rearrangements $\left\{\phi_{n}\right\}$ of the Vilenkin system. For each pair $N, \nu$ with $N=0,1, \ldots, 1 \leqslant \nu<p_{N}$, let $\sigma_{N, \nu}$ be a permutation of the nonnegative integers $\{0,1,2, \ldots\}$ such that $\sigma_{N, \nu}(j)=j$ for all $j \geqslant N$. Let $\psi_{n}^{N, \nu}=\psi_{\sigma_{N, \nu}(n)}$ and let $\left\{\chi_{n}^{N, \nu}\right\}$ denote the standard ordering of $X$ generated by $\left\{\psi_{n}^{N, \nu}\right\}$. Each of the systems $\left(G,\left\{\chi_{n}^{N, \nu}\right\}\right)$ is a particular case of the Vilenkin system studied in [5]. We define the rearrangements $\left\{\phi_{n}\right\}$ as follows: Let $\phi_{0}=\chi_{0}$, and for any integer $n$ with $\nu m_{N} \leqslant n<(\nu+1) m_{N}, N \geqslant 0,1 \leqslant \nu<p_{N}$, we define $\phi_{n}=\chi_{n}^{N, \nu}$. In other words, if $m_{0}^{N, \nu}=1$ and $m_{s}^{N, \nu}=\Pi_{i=0}^{s-1} p_{\sigma_{N, \nu}(i)}$, then for any integer $n$ with $\nu m_{N} \leqslant n<(\nu+1) m_{N}$, write $n=\Sigma_{s=0}^{N} a_{s}^{N, \nu} m_{s}^{N, \nu}$, with $a_{N}^{N, \nu}=\nu, 0 \leqslant a_{s}^{N, \nu}<p_{\sigma_{N, \nu}(s)}$. We have

$$
\phi_{n}=\prod_{s=0}^{N}\left(\psi_{s}^{N, \nu}\right)^{a_{s}^{N, \nu}}=\prod_{s=0}^{N}\left(\psi_{\sigma_{N, \nu}(s)}\right)^{a_{s}^{N, \nu}}
$$

We note that for each $\phi_{n}$ with $\nu m_{N} \leqslant n<(\nu+1) m_{N}$, there is a $\chi_{k}$ with $\nu m_{N} \leqslant$ $k<(\nu+1) m_{N}$ such that $\phi_{n}=\chi_{k}$. Hence $\left\{\phi_{n}\right\}$ is a rearrangement of $\left\{\chi_{n}\right\}$ within blocks of indices $\nu m_{N} \leqslant n<(\nu+1) m_{N}, N \geqslant 0,1 \leqslant \nu<p_{N}$.

If $\sigma_{N, \nu}$ is the identity permutation for all $N, \nu$, then $\left\{\phi_{n}\right\}$ coincides with $\left\{\chi_{n}\right\}$. In the case of the Walsh system, $p_{N}=2$ for all $N$, so that $\nu=1$ for all $N$. The Kaczmarz rearrangement occurs when $\sigma_{N, 1}(j)=N-j-1,0 \leqslant j \leqslant N-1$, $N=1,2, \ldots$.

We will use the following notation. For $f \in L^{1}(G)$, let

$$
T_{n} f=\sum_{j=0}^{n-1} \phi_{j} \int_{G} f \bar{\phi}_{j} d \mu
$$

(respectively $S_{n} f=\sum_{j=0}^{n-1} \chi_{j} \int_{G} f \bar{\chi}_{j} d \mu$ ) be the $n$th partial sum of the Fourier series of $f$ with respect to $\left\{\phi_{n}\right\}$ (respectively $\left\{\chi_{n}\right\}$ ). Since $\left\{\phi_{n}\right\}$ is a rearrangement of $\left\{x_{n}\right\}$ within blocks of indices $\nu m_{N} \leqslant n<(\nu+1) m_{N}, T_{\nu m_{N}} f=S_{\nu m_{N}} f$ for $N \geqslant 0,1 \leqslant \nu<p_{N}$. Let $T^{*} f=\sup _{n \geqslant 1}\left|T_{n} f\right|$, and $S^{*} f=\sup _{n \geqslant 1}\left|S_{n} f\right|$. We have the following

THEOREM 1. There are absolute constants $C$ and $C_{q}$ such that

(a) $\left\|T^{*} f\right\|_{q} \leqslant C_{q}\|f\|_{q}, f \in L^{q}(G), 2 \leqslant q<\infty$,

(b) $\mu\left\{x \in G: T^{*} f(x)>\lambda\right\} \leqslant C \exp \left(-C N\|f\|_{\infty}\right), \lambda>0, f \in L^{\infty}(G)$.

This implies the a.e. $(\mu)$ convergence of $T_{n} f$ to $f$ for $f \in L^{q}(G), 2 \leqslant q \leqslant \infty$. To establish the $L^{q}$ result for $1<q<2$, we have to restrict ourselves to 
the subclass of rearrangements given by permutations $\left\{\sigma_{N, \nu}\right\}$ that satisfy the "block condition":

For each pair $N, \nu$ with $N=1,2, \ldots, 1 \leqslant \nu<p_{N}$, and for each $0 \leqslant j \leqslant$ $N-1$, there is an integer $k_{N, \nu, j}$, with $0 \leqslant k_{N, \nu, j} \leqslant N-j-1$, such that

$$
\left\{\sigma_{N, \nu}(0), \ldots, \sigma_{N, \nu}(j)\right\}=\left\{k_{N, \nu, j}, k_{N, \nu, j}+1, \ldots, k_{N, \nu, j}+j\right\} .
$$

This condition is only used in the proof of Lemma 1. We remark that this subclass also contains the identity rearrangement given by $\sigma_{N, \nu}(j)=j, 0 \leqslant j \leqslant N-1$, as well as the Kaczmarz rearrangement given by $\sigma_{N, 1}(j)=N-j-1,0 \leqslant j \leqslant$ $N-1$, in the case of the Walsh system.

THEOREM 2. If $\left\{\sigma_{N, \nu}\right\}$ satisfies the block condition, then there are absolute constants $C$ and $C_{q}$ such that:

(a) $\left\|T^{*} f\right\|_{q} \leqslant C_{q}\|f\|_{q}, f \in L^{q}(G), 1<q<2$.

(b) $\left\|T^{*} f\right\|_{1} \leqslant C \int_{G}|f|\left(\log ^{+}|f|\right)^{3} d \mu+C, f \in L\left(\log ^{+} L\right)^{3}(G)$.

(c) If $\int_{G}|f|\left(\log ^{+}|f|\right)^{2} \log ^{+} \log ^{+}|f| d \mu<\infty$, then $T_{n} f$ converges to $f$ a.e. $(\mu)$.

The absolute constants $C$ and $C_{q}$ in the above theorems are independent of the permutations $\left\{\sigma_{N, \nu}\right\}$.

REMARKS. It might be interesting to compare our a.e. convergence results with those of other systems.

For the usual Vilenkin-Fourier series, the proof of [5] yields the following result (see [6], [10] and [8]). There are absolute constants $C$ and $C_{q}$ such that:

(a) $\left\|S^{*}\right\|_{q} \leqslant C_{q}\|f\|_{q}, f \in L^{q}(G), 1<q<\infty$.

(b) $\mu\left\{x \in G: S^{*} f(x)>\lambda\right\} \leqslant C \exp \left(-C \lambda\|f\|_{\infty}\right), \lambda>0, f \in L^{\infty}(G)$.

(c) $\left\|S^{*} f\right\|_{1} \leqslant C \int_{G}|f|\left(\log ^{+}|f|\right)^{2} d \mu+C, f \in L\left(\log ^{+} L\right)^{2}(G)$.

(d) If $\int_{G}|f|\left(\log ^{+}|f|\right) \log ^{+} \log ^{+}|f| d \mu<\infty$, then $S_{n} f$ converges to $f$ a.e. $(\mu)$.

For the Kaczmarz rearrangement of the Walsh system, it is shown in [16] that if $\int_{0}^{1}|f|\left(\log ^{+}|f|\right)^{2} d x<\infty$, then the partial sums of the Walsh-KaczmarzFourier series of $f$ converge a.e. to $f$. This is proved by using a result of $\mathrm{P}$. Sjolin [10] on the a.e. convergence of Walsh-Fourier series.

As for a.e. divergence results, it is known that there exist functions in $L\left(\log ^{+} \log ^{+} L\right)^{1-\epsilon}, 0<\epsilon<1$, whose Walsh-Fourier series diverge a.e. (See Moon [9].) In the case of the Walsh-Kaczmarz-Fourier series, such a result holds for the Orlicz class $L\left(\log ^{+} L\right)^{1-\epsilon}, 0<\epsilon<1$. (See Balashov [1].)

3. Outline of proof. The proof will proceed as follows. The $L^{2}$ result (the case $q=2$ of Theorem 1 part (a)) will be obtained as a consequence of the corresponding result for the Vilenkin-Fourier series [5]. This will be proved in $\S 4$. 
To prove the remainder of Theorems 1 and 2, it is enough to prove the following basic result:

We call $f$ a special function if $f=h I_{F}$ where $I_{F}$ is the characteristic function of a measurable subset $F$ of $G$, and $h$ a measurable function with values in $(1 / 2,1]$.

Basic result. Let $1<q<\infty$. For $1<q<2$ we assume that the permutations $\left\{\phi_{N, \nu}\right\}$ satisfy the block condition. Then there are absolute constants $C$ and $C_{q}$ such that

$$
\mu\left\{x \in G: T^{*} f>\lambda\right\} \leqslant C_{q}^{q} \lambda^{-q} \int_{G}|f|^{q} d \mu
$$

where $\lambda>0, f$ is a special function, and $C_{q} \leqslant C(q-1)^{-2}$ if $1<q<2$ and $C_{q} \leqslant C q$ if $2 \leqslant q<\infty$.

The basic result together with the theory of interpolation and extrapolation of operators imply the remainder of Theorems 1 and 2. The proof of this implication can be found in Hunt [6], Sjolin [10], and Hunt and Taibleson [8]. We will omit the proof.

The argument used in the $L^{2}$ proof will also be used to reduce the basic result to a basic lemma. The statement of the lemma and the reduction will be given in $\S 4$.

In order to prove the basic lemma we need estimates on certain maximal functions of the Hardy-Littlewood type. These estimates are obtained in $\S 5$.

The remainder of this chapter will be devoted to the proof of the basic lemma. The techniques used are modifications of those in [5], which are adaptations of the methods of L. Carleson [3] and R. A. Hunt [6], [7]. We will emphasize the difference between our proof and that for the usual Vilenkin-Fourier series [5]. The parts which are essentially the same will be omitted.

4. Proof of the $L^{2}$ result and reduction of the basic result to the basic lemma. For $N=0,1, \ldots, 1 \leqslant \nu<p_{N}$, let

$$
f^{N, \nu}=S_{(\nu+1) m_{N}} f-S_{\nu m_{N}} f=T_{(\nu+1) m_{N}} f-T_{\nu m_{N}} f,
$$

and let $S_{n}^{N, \nu} g$ denote the $n$th partial sum of $g \in L^{1}(G)$ with respect to the system $\left\{\chi_{n}^{N, \nu}\right\}$. Then, for $\nu m_{N} \leqslant n<(\nu+1) m_{N}$,

$$
T_{n} f=S_{n}^{N, \nu} f^{N, \nu}+T_{\nu m_{N}} f=S_{n}^{N, \nu} f^{N, \nu}+S_{\nu m_{N}} f .
$$

Hence, for any $f \in L^{1}(G)$, 


$$
\begin{aligned}
T^{*} f= & \sup _{n \geqslant 1}\left|T_{n} f\right| \\
\leqslant & \sup _{N \geqslant 1} \sup _{1 \leqslant \nu<p_{N}} \sup _{\nu m_{N} \leqslant n<(\nu+1) m_{N}}\left|S_{n}^{N, \nu} f^{N, \nu}\right| \\
& +\sup _{N \geqslant 0} \sup _{1 \leqslant \nu<p_{N}}\left|S_{\nu m_{N}} f\right| \\
\leqslant & \sup _{N \geqslant 1} \sup _{1 \leqslant \nu<p_{N}} \sup _{N} \leqslant n<(\nu+1) m_{N} \mid S_{n}^{N, \nu} f^{N, \nu \mid} \\
& +S^{*} f .
\end{aligned}
$$

We now prove Theorem 1 part (a) for the case $q=2$. It follows from [5] that the $L^{2}$ result holds uniformly for each of the systems $\left\{\chi_{n}\right\}$ and $\left\{\chi_{n}^{N, \nu}\right\}$, $N=1,2, \ldots, 1 \leqslant \nu<p_{N}$. Hence we obtain

$$
\int_{G}\left|S^{*} f\right|^{2} d \mu \leqslant C \int_{G}|f|^{2} d \mu,
$$

and

$$
\int_{G} \sup _{\nu m_{N} \leqslant n<(\nu+1) m_{N}}\left|S_{n}^{N, \nu} f^{N, \nu}\right|^{2} d \mu \leqslant C \int_{G}\left|f^{N, \nu}\right|^{2} d \mu,
$$

where $C$ is independent of $N$ and $\nu$. Thus

$$
\begin{aligned}
\int_{G} \sup _{N \geqslant 1} \sup _{1<\nu<p_{N}} \sup _{\nu m_{N} \leqslant n<(\nu+1) m_{N}}\left|S_{n}^{N, \nu} f^{N, \nu}\right|^{2} d \mu \\
\quad \leqslant \sum_{N=1}^{\infty} \sum_{\nu=1}^{p_{N}-1} \int_{G} \sup _{\nu m_{N} \leqslant n<(\nu+1) m_{N}}\left|S_{n}^{N, \nu} f^{N, \nu}\right|^{2} d \mu \\
\quad \leqslant C \sum_{N=1}^{\infty} \sum_{\nu=1}^{p_{N}-1} \int_{G}\left|f^{N, \nu}\right|^{2} d \mu \\
\quad \leqslant C \int_{G} \mid f^{2} d \mu,
\end{aligned}
$$

by Plancherel's formula. These estimates together with (2) yield the $L^{2}$ result.

We also use (2) to reduce the basic result to a basic lemma.

Let $f$ be a special function. From [5], we have

$$
\mu\left\{x \in G: S^{*} f(x)>\lambda\right\} \leqslant C_{q}^{q} \lambda^{-q} \int_{G}|f|^{q} d \mu,
$$

with $C_{q} \leqslant C q^{2}(q-1)^{-1}, 1<q<\infty$. (The proof in [5] is for characteristic functions, and can be carried out for special functions with very little change.) Thus, in view of (2), the basic result follows immediately from the

BASIC LEMMA. Let $1<q<\infty$. For the case $1<q<2$, we assume that the permutations $\left\{\sigma_{N, \nu}\right\}$ satisfy the block condition. Fix $\lambda$ and $f$ with $\lambda>0$ 
and $f$ a special function. Let $L=L(q)=\left[4 q^{2} /(q-1)\right]+1$, where $[\cdot]$ denotes the greatest integer function. Then there exist an exceptional set $E=E(\lambda, q, f)$ in $G$, and absolute constants $C$ and $C_{q}$ with $C_{q} \leqslant C q /(q-1)$, such that $\mu(E) \leqslant$ $C_{q}^{q} \lambda^{-q} \int_{G}|f|^{q} d \mu$, and $x \in G \sim E, N \geqslant 1,1 \leqslant \nu<p_{N}, \nu m_{N} \leqslant n<(\nu+1) m_{N}$ implies $\left|S_{n}^{N, v} f^{N, \nu}\right| \leqslant C L \lambda$.

The idea of proof of the basic lemma is as follows: For each $N=1$, $2, \ldots, 1 \leqslant \nu<p_{N}$, we use the techniques of L. Carleson [3] and R. A. Hunt [6], [7] to construct an exceptional set $E_{N, \nu}$ such that if $x \notin E_{N, \nu}$, then $\left|S_{n}^{N, \nu} f^{N, \nu}(x)\right| \leqslant C L \lambda$ for $\nu m_{N} \leqslant n<(\nu+1) m_{N}$. Our exceptional set $E$ will be the union of all the exceptional sets $E_{N, \nu}$. The lemmas proved in the next section will be essential for estimating $\mu(E)$.

REMARK. In the case of the Walsh functions, the sequence $\left\{\psi_{n}\right\}$ is the Rademacher sequence $\left\{r_{n}\right\}$. It was observed in [13], that if $\left\{r_{n_{j}}\right\}$ is any rearrangement of $\left\{r_{n}\right\}$, then the $L^{q}$ result, $1<q \leqslant \infty$, holds for the system generated by $\left\{r_{n_{j}}\right\}$ and the standard ordering. This result uses the $L^{q}$ result for the Walsh system with the standard ordering and the existence of a bijective measure-preserving transformation $\tau$ such that $r_{n_{i}}=r_{i} \circ \tau$ for $i \geqslant 0$. In the context of Vilenkin systems, there exists a bijective measure-preserving transformation $\tau: \prod_{i=0}^{\infty} Z_{p_{i}} \rightarrow$ $\Pi_{i=0}^{\infty} Z_{p_{\sigma(i)}}$ such that $\psi_{\sigma(i)}=\psi_{i}^{\prime} \circ \tau$ where $\left\{\psi_{i}^{\prime}\right\}_{i=0}^{\infty}$ is the sequence for $\Pi_{i=0}^{\infty} Z_{p_{\sigma(i)}}$ corresponding to the Rademacher functions. This fact and the result of [5] can be used to construct exceptional sets $\widetilde{E}_{N, \nu}$ which are similar to the sets $E_{N, \nu}$ we construct in this work. Because we want to estimate the size of the union of all the sets $E_{N, \nu}$, our sets $E_{N, \nu}$ are in fact different than the sets $\widetilde{E}_{N, \nu}$ we would obtain above. Our construction of the exceptional sets is a modification of the Carleson-Hunt technique used in [5], and we shall be brief on those parts of the construction which are essentially unchanged.

5. Maximal inequalities of the Hardy-Littlewood type. Let $E\left(g \mid g_{1}, \ldots, g_{n}\right)$ denote the conditional expectation of $g$ given the Borel field generated by $g_{1}$, $\ldots, g_{n}$. To prove the basic lemma for the case $1<q<2$, we need the weak type $(r, r)(r>1)$ estimate of the operator

$$
g \rightarrow g^{*}=\sup _{\substack{0 \leqslant j<N ; 1 \leqslant \nu<p_{N} \\ N \geqslant 1}} E\left(|g| \mid \psi_{0}^{N, \nu}, \ldots, \psi_{j}^{N, \nu}\right) .
$$

Note that for the case where $\sigma_{N, \nu}$ is the identity permutation for all $N, \nu, g^{*}$ is just the martingale maximal function $\sup _{j \geqslant 0} E\left(|g| \mid \psi_{0}, \ldots, \psi_{j}\right)$.

Lemma 1. Suppose $\left\{\sigma_{N, \nu}\right\}$ satisfies the block condition. Then, for $1<$ $r<\infty$, 


$$
\mu\left\{x \in G: g^{*}(x)>\lambda\right\} \leqslant B_{r}^{r} \lambda^{-r} \int_{G}|g|^{r} d \mu,
$$

where $\lambda>0, g \in L^{r}(G)$, and $B_{r} \leqslant r /(r-1)$.

In view of (1), this is a corollary of

LEMMA 2. For $1<r<\infty$,

$$
m\left\{x \in G: \sup _{n, k \geqslant 0} E\left(|g| \mid \psi_{n}, \ldots, \psi_{n+k}\right)(x)>\lambda\right\} \leqslant B_{r}^{r} \lambda^{-r} \int_{G}|g|^{r} d \mu,
$$

where $\lambda>0, g \in L^{r}(G)$, and $B_{r} \leqslant r /(r-1)$.

Proof. We can assume that $g \geqslant 0$. We begin by observing that for any $n$, $k \geqslant 0$

(3) $E\left(g \mid \psi_{n}, \ldots, \psi_{n+k}\right)=E\left(E\left(g \mid \psi_{0}, \ldots, \psi_{n+k}\right) \mid \psi_{n}, \psi_{n+1}, \ldots\right)$.

This is a consequence of the independence of the functions $\left\{\psi_{n}\right\}$, and the following fact (see, for example, $[4$, p. 285]):

Suppose $F_{1}, F_{2}, F_{3}$ are three Borel fields such that $F_{1} \vee F_{2}$, the Borel field generated by $F_{1} \cup F_{2}$, is independent of $F_{3}$. Then, for each integrable, $F_{1}$ measurable function $h$, we have $E\left(h \mid F_{2}\right)=E\left(h \mid F_{2} \vee F_{3}\right)$.

With $F_{1}=F\left(\psi_{0}, \ldots, \psi_{n+k}\right), F_{2}=F\left(\psi_{n}, \ldots, \psi_{n+k}\right), F_{3}=$ $F\left(\psi_{n+k+1}, \psi_{n+k+2}, \ldots\right)$ and $h=E\left(g \mid \psi_{0}, \ldots, \psi_{n+k}\right)$, we obtain

$$
\begin{aligned}
& E\left(E\left(g \mid \psi_{0}, \ldots, \psi_{n+k}\right) \mid \psi_{n}, \psi_{n+1}, \ldots\right) \\
& \quad=E\left(E\left(g \mid \psi_{0}, \ldots, \psi_{n+k}\right) \mid \psi_{n}, \ldots, \psi_{n+k}\right)=E\left(g \mid \psi_{n}, \ldots, \psi_{n+k}\right),
\end{aligned}
$$

which is (3).

The rest of the proof consists of repeated applications of the martingale maximal theorem [11, p. 91]:

$$
\begin{aligned}
\mu\{x \in G: & \left.\sup _{n, k \geqslant 0} E\left(g \mid \psi_{n}, \ldots, \psi_{n+k}\right)(x)>\lambda\right\} \\
& =\mu\left\{x \in G: \sup _{n, k \geqslant 0} E\left(E\left(g \mid \psi_{0}, \ldots, \psi_{n+k}\right) \mid \psi_{n}, \psi_{n+1}, \ldots\right)(x)>\lambda\right\} \\
& \leqslant \mu\left\{x \in G: \sup _{n \geqslant 0} E\left(\sup _{k \geqslant 0} E\left(g \mid \psi_{0}, \ldots, \psi_{k}\right) \mid \psi_{n}, \psi_{n+1}, \ldots\right)(x)>\lambda\right\} \\
& \leqslant \lambda^{-r} \int_{G} \sup _{k \geqslant 0}\left(E\left(g \mid \psi_{0}, \ldots, \psi_{k}\right)\right)^{r} d \mu \\
& \leqslant B_{r}^{r} \lambda^{-r} \int_{G}|g|^{r} d \mu,
\end{aligned}
$$


where $B_{r} \leqslant r /(r-1)$. This completes the proof of Lemma 2 .

REMARK 1. We remark that the mapping $g \rightarrow \sup _{n, k \geqslant 0} E\left(|g| \mid \psi_{n}, \ldots, \psi_{n+k}\right)$ is not of weak type $(1,1)$. Consequently, for $r=1$, the conclusion of Lemma 1 would not hold in general. This accounts for the fact that the argument we use only enables us to establish the a.e. convergence result for the rearranged series for functions in the class $L\left(\log ^{+} L\right)^{2} \log ^{+} \log ^{+} L$, whereas, for the Vilenkin-Fourier series, a similar argument yields the same result for functions in the class $L\left(\log ^{+} L\right) \log ^{+} \log ^{+} L$.

The following is a modification of an example communicated to us by $\mathrm{K} . \mathrm{H}$. Moon. We assume, without loss of generality, that $p_{i} \geqslant 2, i=0,1, \ldots$ We will construct a sequence of functions $\left\{g_{m}\right\}, 0 \leqslant g_{m} \in L^{1}(G)$, such that

$$
\mu\left\{x \in G: \sup _{n, k \geqslant 0} E\left(g_{m} \mid \psi_{n}, \ldots, \psi_{n+k}\right)(x)>1 / 2\right\} \geqslant 1 / 2, \quad m=1,2, \ldots,
$$

but

$$
\int_{G}\left|g_{m}\right| d \mu \rightarrow 0 \text { as } m \rightarrow \infty \text {. }
$$

For each $m=1,2, \ldots, j=0,1, \ldots$, let $A_{m, j}=\left\{x G: \psi_{m k}(x)=\right.$ $\left.\psi_{m j+1}(x)=\ldots=\psi_{m j+m-1}(x)=1\right\}$, the annihilator of the subgroup generated by $\psi_{m j}, \ldots, \psi_{m j+m-1}$. Note that $\mu\left(A_{m, j}\right)=\Pi_{i=m j}^{m j+m-1} p_{i}^{-1} \geqslant p^{-m}$. Since, for each $m,\left\{A_{m, j}\right\}_{j=1}^{\infty}$ are independent, and $\Sigma_{j=0}^{\infty} \mu\left(A_{m, j}\right) \geqslant \Sigma_{j=0}^{\infty} p^{-m}=\infty$, we have, by the Borel-Cantelli lemma, $\mu\left(\bigcup_{j=0}^{\infty} A_{m, j}\right)=1$ for every $m$. Therefore, there exists $J_{m}$ such that

$$
\mu\left(\bigcup_{j=0}^{J_{m}-1} A_{m, j}\right) \geqslant 1 / 2 .
$$

Let $A_{m}=\bigcap_{j=0}^{J_{m}-1} A_{m, j}$. We have $\mu\left(A_{m}\right)=\Pi_{i=0}^{m J_{m}-1} p_{i}^{-1}$. For $m=1,2, \ldots$, define

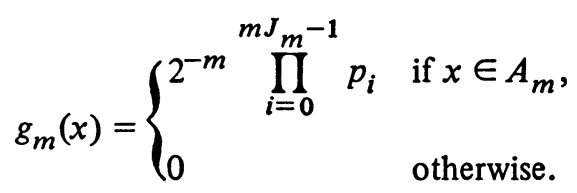

Then, for $x \in A_{m, j}, j=0,1, \ldots, J_{m}-1$,

$$
\begin{aligned}
E\left(g_{m} \mid \psi_{m j}, \ldots, \psi_{m j+m-1}\right)(x) & =\mu\left(A_{m, j}\right)^{-1} \int_{A_{m, j}} g_{m} d \mu \\
& =\left(\prod_{i=m j}^{m j+m-1} p_{i}\right) 2^{-m} \geqslant 1 .
\end{aligned}
$$


Thus we have

$$
\begin{aligned}
\mu\{x \in G: & \left.\sup _{n, k \geqslant 0} E\left(g_{m} \mid \psi_{n}, \ldots, \psi_{n+k}\right)(x)>1 / 2\right\} \\
& \geqslant \mu\left(\bigcup_{j=0}^{J_{m}^{-1}} A_{m, j}\right) \geqslant 1 / 2,
\end{aligned}
$$

but

$$
\int_{G}\left|g_{m}\right| d \mu=2^{-m} \rightarrow 0 \text { as } m \rightarrow \infty \text {. }
$$

This shows that $g \rightarrow \sup _{n, k \geqslant 0} E\left(|g| \mid \psi_{n}, \ldots, \psi_{n+k}\right)$ is not of weak type $(1,1)$.

REMARK 2. If we relaxed the block condition on the permutations $\left\{\sigma_{N, \nu}\right\}$, Lemma 1 would be false in general for any $r \geqslant 1$.

We will show that the operator

$$
g \rightarrow \sup _{0<j<n ; n \geqslant 1} E\left(|g| \psi_{0}, \ldots, \psi_{j-1}, \psi_{j+1}, \ldots, \psi_{n}\right)
$$

is not of weak type $(r, r)$ for any $r \geqslant 1$. Again we assume $p_{i} \geqslant 2, i=0,1, \ldots$. Let $A_{m}=A\left(\psi_{0}, \ldots, \psi_{m}\right)$, and, for $0 \leqslant j<m$,

$$
A_{m, j}=A\left(\psi_{0}, \ldots, \psi_{j-1}, \psi_{j+1}, \ldots, \psi_{m}\right) .
$$

We observe that each $A_{m, j}$ is the union of $A_{m}$ and $p_{j}-1$ other cosets of $A_{m}$. Also $A_{m, j_{1}} \cap A_{m, j_{2}}=A_{m}$ for $j_{1} \neq j_{2}$. Thus

$$
\mu\left(\bigcup_{j=0}^{m-1} A_{m, j}\right)=\left(\sum_{j=0}^{m-1}\left(p_{j}-1\right)+1\right) \prod_{i=0}^{m} p_{i}^{-1} \geqslant(m+1) \prod_{i=0}^{m} p_{i}^{-1} .
$$

For $m=1,2, \ldots$, define

$$
g_{m}(x)= \begin{cases}1 & \text { if } x \in A_{m} \\ 0 & \text { otherwise }\end{cases}
$$

Then for $x \in A_{m, j}$,

$$
\begin{aligned}
E\left(g_{m} \mid \psi_{0}, \ldots, \psi_{j-1}, \psi_{j+1}, \ldots, \psi_{m}\right)(x) & =\mu\left(A_{m, j}\right)^{-1} \int_{A_{m, j}} g_{m} d \mu \\
& =p_{j}^{-1} \geqslant p^{-1}
\end{aligned}
$$

Therefore,

$$
\begin{gathered}
\mu\left\{x \in G: \sup _{0<j<n ; n \geqslant 1} E\left(g_{m} \mid \psi_{0}, \ldots, \psi_{j-1}, \psi_{j+1}, \ldots, \psi_{n}\right)(x)>(2 p)^{-1}\right\} \\
\geqslant \mu\left(\bigcup_{j=0}^{m-1} A_{m, j}\right) \geqslant(m+1) \prod_{i=0}^{m} p_{i}^{-1} .
\end{gathered}
$$


On the other hand, for any $r \geqslant 1, \int_{G}\left|g_{m}\right|^{r} d \mu=\Pi_{i=0}^{m} p_{i}^{-1}$. This verifies our statement.

To prove the basic lemma for the case $2 \leqslant q<\infty$, it is sufficient to consider the operator

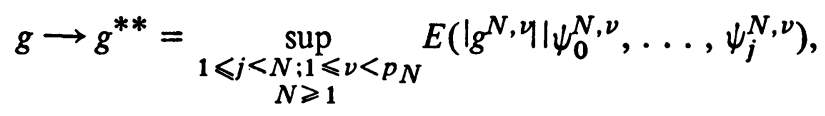

where, as we recall, $g^{N, \nu}=S_{(\nu+1) m_{N}} g-S_{\nu m_{N}} g$.

LEMma 3. For $2 \leqslant r \leqslant \infty,\left\|g^{* *}\right\|_{r} \leqslant 2\|g\|_{r}, g \in L^{r}(G)$. Consequently, we have

$$
\mu\left\{x \in G: g^{* *}(x)>\lambda\right\} \leqslant 2^{r} \lambda^{-r} \int_{G}|g|^{r} d \mu,
$$

where $\lambda>0, g \in L^{r}(G)$.

PROOF. For $r=2$,

$$
\int_{G}\left|g^{* *}\right|^{2} d \mu \leqslant \sum_{N=1}^{\infty} \sum_{\nu=1}^{p_{N^{-1}}} \int_{G} \sup _{1 \leqslant j<N}\left(E\left(\left|g^{N, \nu}\right| \mid \psi_{0}^{N, \nu}, \ldots, \psi_{j}^{N, \nu}\right)\right)^{2} d \mu
$$

$$
\begin{aligned}
& \leqslant 4 \sum_{N=1}^{\infty} \sum_{\nu=1}^{p_{N}^{-1}} \int_{G} \mid g^{N,\left.\nu\right|^{2}} d \mu \\
& \leqslant 4 \int_{G}|g|^{2} d \mu,
\end{aligned}
$$

by the martingale maximal theorem [11, p. 91] and Plancherel's formula.

For $r=\infty$, since $g^{N, \nu}=\psi_{N}^{\nu} S_{m_{N}}\left(\bar{\psi}_{N}^{\nu} g\right)$, and, for any $h \in L^{1}(G), S_{m_{N}}(h)=$ $E\left(h \mid \psi_{0}, \ldots, \psi_{N-1}\right)($ see $[5,(15)])$, we have $\left\|g^{N, \nu}\right\|_{\infty} \leqslant\|g\|_{\infty}$. Therefore,

$$
\left\|g^{* *}\right\|_{\infty} \leqslant\|g\|_{\infty} .
$$

Our lemma follows from (4) and (5) and the Riesz convexity theorem (Calderón and Zygmund [2]).

6. Proof of the basic lemma for the case $1<q<2$. We will first construct, for each $N \geqslant 1,1 \leqslant \nu<p_{N}$ an exceptional set $E_{N, \nu}$ such that, if $x \notin E_{N, \nu}$, then $\left|S_{n}^{N, \nu} f^{N, \nu}(x)\right| \leqslant C L \lambda$ for $\nu m_{N} \leqslant n<(\nu+1) m_{N}$. Many of the details will be omitted since they are essentially the same as those in [5].

We will use the following notation. For $N \geqslant 1,1 \leqslant \nu<p_{N}$, let $G_{0}^{N, \nu}=G$, and for $j=1, \ldots, N+1$, let $G_{j}^{N, \nu}=A\left(\psi_{0}^{N, \nu}, \ldots, \psi_{j-1}^{N, \nu}\right)$, the annihilator of the subgroup generated by $\psi_{0}^{N, \nu}, \ldots, \psi_{j-1}^{N, \nu} \cdot \omega_{N, \nu, j}$ will denote any coset of $G_{j}^{N, \nu}$. For $\omega_{N, \nu, j}$ fixed with $j \geqslant 1$, let $\omega_{N, \nu, j}^{*}$ denote the unique coset of $G_{j-1}^{N, \nu}$ 
which contains $\omega_{N, v, j}$. Note that $\mu\left(\omega_{N, v, j}^{*}\right) \leqslant p \mu\left(\omega_{N, v, j}\right)$ for all $N, \nu, j$.

Fix $N \geqslant 1,1 \leqslant \nu<p_{N}$ and let $\nu m_{N} \leqslant n<(\nu+1) m_{N}$. Given $\omega_{N, \nu, j}$, $1 \leqslant j \leqslant N+1$, we define the "coefficients" $a_{n}\left(\omega_{N, \nu, j}\right)$ as

$$
\begin{aligned}
a_{n}\left(\omega_{N, \nu, j}\right) & =\mu\left(\omega_{N, \nu, j}\right)^{-1} \int_{\omega_{N, v, j}} f^{N, \nu} \overline{\chi_{n}^{N, \nu}} d \mu \\
& =E\left(f^{N, \nu} \overline{\chi_{n}^{N, \nu}} \mid \psi_{0}^{N, \nu}, \ldots, \psi_{j-1}^{N, \nu}\right)(x),
\end{aligned}
$$

where $x \in \omega_{N, \nu, j}$. We also write

$$
A_{n}\left(\omega_{N, \nu, j}^{*}\right)=\max \left\{\left|a_{n}\left(\bar{\omega}_{N, \nu, j}\right)\right|: \bar{\omega}_{N, \nu, j}^{*}=\omega_{N, \nu, j}^{*}\right\} .
$$

Finally, for $\nu m_{N} \leqslant n<(\nu+1) m_{N}, N \geqslant 1,1 \leqslant \nu<p_{N}$, write $n=$ $\Sigma_{s=0}^{N} a_{s}^{N, v} m_{s}^{N, \nu}$. Fix $\omega_{N, \nu, j}$ with $0 \leqslant j \leqslant N$. We define

$$
n\left(\omega_{N, \nu, j}\right)=\sum_{s=j}^{N} a_{s}^{N, \nu} m_{s}^{N, \nu}
$$

We observe that

$$
\left|a_{n\left(\omega_{N, \nu, j}\right)}\left(\omega_{N, \nu, j}\right)\right|=\left|a_{n}\left(\omega_{N, \nu, j}\right)\right| .
$$

The first part $V_{N, \nu}$ of the exceptional set $E_{N, \nu}$ is used to control the numbers $A_{n}\left(\omega_{N, v, j}\right)$. Let

$$
V_{N, \nu}=\bigcup_{j=1}^{N+1}\left\{\omega_{N, \nu, j}^{*}: \mu\left(\omega_{N, \nu, j}\right)^{-1} \int_{\omega_{N, \nu, j} \mid f^{(q+1) / 2}} d \mu \geqslant \lambda^{(q+1) / 2}\right\} .
$$

Then

$$
\omega_{N, \nu, j}^{*} \not \subset V_{N, \nu} \text { implies } A_{n}\left(\omega_{N, \nu}^{*}\right)<\lambda,
$$

for all $1 \leqslant j \leqslant N+1$ and $\nu m_{N} \leqslant n \leqslant(\nu+1) m_{N}$. This can be seen as follows. If $\bar{\omega}_{N, \nu, j}^{*}=\omega_{N, \nu, j}^{*}$ and $x \in \bar{\omega}_{N, \nu, j}$, we have

$$
\begin{aligned}
\left|a_{n}\left(\bar{\omega}_{N, \nu, j}\right)\right| & \leqslant\left(E\left(\left|f^{N, \nu \mid(q+1) / 2}\right| \psi_{0}^{N, \nu}, \ldots, \psi_{j-1}^{N, \nu}\right)(x)\right)^{2 /(q+1)} \\
& \leqslant\left(E\left(E\left(|f|^{(q+1) / 2} \mid \psi_{0}, \ldots, \psi_{N-1}\right) \mid \psi_{0}^{N, \nu}, \ldots, \psi_{j-1}^{N, \nu}\right)(x)\right)^{2 /(q+1)} \\
& =\left(E\left(\left|f^{(q+1) / 2}\right| \psi_{0}^{N, \nu}, \ldots, \psi_{j-1}^{N, \nu}\right)(x)\right)^{2 /(q+1)} \\
& =\left(\mu\left(\bar{\omega}_{N, \nu, j}\right)^{-1} \int_{\left.\bar{\omega}_{N, \nu, j}|f|^{(q+1) / 2} d \mu\right)^{2 /(q+1)}}\right. \\
& <\lambda,
\end{aligned}
$$

since $\bar{\omega}_{N, \nu, j}^{*} \not \subset V_{N, \nu}$. 
For each positive integer $k$, let $\Lambda_{k}(N, \nu)$ be the collection of pairs $\left(n\left(\omega_{N, \nu, j}\right), \omega_{N, \nu, j}\right)$ such that $\nu m_{N} \leqslant n<(\nu+1) m_{N}$ and

(7)(i) $\left|a_{n\left(\omega_{N, \nu, j}\right)}\left(\omega_{N, \nu, j}\right)\right| \geqslant p^{-k} \lambda$, and

(ii) either $j=1$ and $\left|a_{n\left(\omega_{N, \nu, j}\right)}\left(\omega_{N, \nu, j}\right)\right|<p^{-k+1} \lambda$, or $1<j \leqslant N$ and $\left|a_{\bar{n}\left(\omega_{N, \nu, \bar{j}}\right)}\left(\omega_{N, \nu, \bar{j}}\right)\right|<p^{-k} \lambda$ for all $\omega_{N, \nu, \bar{j}} \supsetneq \omega_{N, \nu, j}$ and $\bar{n}$ with $\bar{n}\left(\omega_{N, \nu, j}\right)=$ $n\left(\omega_{N, \nu, j}\right)$.

Let $\Lambda_{k}^{*}(N, \nu)$ be the collection of all pairs $\left(n\left(\omega_{N, \nu, j}^{*}\right), \omega_{N, \nu, j}^{*}\right)$ such that $\left(n\left(\omega_{N, \nu, j}\right), \omega_{N, \nu, j}\right) \in \Lambda_{k}(N, \nu)$ and $\omega_{N, \nu, j}^{*} \not \subset V_{N, \nu}$.

From (7) we have $A_{n\left(\omega_{N, \nu, j}^{*}\right)}\left(\omega_{N, \nu, j}^{*}\right)<p^{-k+1} \lambda$ if $\left(n\left(\omega_{N, \nu, j}^{*}\right), \omega_{N, \nu, j}^{*}\right) \in$ $\Lambda_{k}^{*}(N, v)$ and $j>1$. (See $[5,(54)]$.) If $j=1$, the pair $\left(n\left(\omega_{N, \nu, j}^{*}\right), \omega_{N, \nu, j}^{*}\right)$ may belong to several $\Lambda_{k}^{*}(N, \nu)$ 's. In this case we delete $\left(n\left(\omega_{N, \nu, j}^{*}\right), \omega_{N, \nu, j}^{*}\right)$ from all $\Lambda_{k}(N, \nu)$ 's except for the one with $k$ minimal. Thus each pair $\left(n\left(\omega_{N, \nu, j}^{*}\right), \omega_{N, \nu, j}^{*}\right)$ belongs to at most one $\Lambda_{k}^{*}(N, \nu)$, and $A_{n\left(\omega_{N, \nu, j}^{*}\right)}\left(\omega_{N, \nu, j}^{*}\right)<p^{-k+1} \lambda$. This guarantees that the partition below is well defined.

If $\left(n\left(\omega_{N, \nu, j}^{*}\right), \omega_{N, \nu, j}^{*}\right) \in \Lambda_{k}^{*}(N, \nu)$, we construct a partition $\Pi_{N, \nu}=$ $\Pi_{N, \nu}\left(n\left(\omega_{N, \nu, j}^{*}\right), \omega_{N, \nu, j}^{*}, k\right)$ of $\omega_{N, \nu, j}^{*}$ as follows: Let

$$
\begin{aligned}
& \Omega_{0}=\left\{\bar{\omega}_{N, \nu, j}: \bar{\omega}_{N, \nu, j}^{*}=\omega_{N, \nu, j}^{*}, A_{n\left(\omega_{N, \nu, j}^{*}\right)}\left(\bar{\omega}_{N, \nu, j}\right) \geqslant p^{-k+1} \lambda\right\}, \\
& \Omega_{1}=\left\{\bar{\omega}_{N, \nu, j+1}: \bar{\omega}_{N, \nu, j+1} \subset \omega_{N, \nu, j}^{*} \backslash \Omega_{0}, A_{n\left(\omega_{N, \nu, j}^{*}\right)}\left(\bar{\omega}_{N, \nu, j+1}\right) \geqslant p^{-k+1} \lambda\right\}, \\
& \Omega_{N-j-1}=\left\{\bar{\omega}_{N, \nu, N-1}: \bar{\omega}_{N, \nu, N-1} \subset \omega_{N, \nu, j}^{*} \bigcup_{s=0}^{N-j-2} \Omega_{s},\right. \\
&\left.A_{n\left(\omega_{N, \nu, j}^{*}\right)}\left(\bar{\omega}_{N, \nu, N-1}\right) \geqslant p^{-k+1} \lambda\right\},
\end{aligned}
$$

and

$$
\Omega_{N-j}=\left\{\bar{\omega}_{N, \nu, N}: \bar{\omega}_{N, \nu, N} \subset \omega_{N, \nu, j}^{*} \backslash \bigcup_{s=0}^{N-j-1} \Omega_{s}\right\} .
$$

Then $\Pi_{N, \nu}\left(n\left(\omega_{N, \nu, j}^{*}\right), \omega_{N, \nu, j}^{*}, k\right)=\bigcup_{s=0}^{N-j} \Omega_{s}$ is a partition of $\omega_{N, \nu, j}^{*}$. For $\bar{\omega} \in$ $\Pi_{N, \nu}\left(n\left(\omega_{N, \nu, j}^{*}\right), \omega_{N, \nu, j}^{*}, k\right)$, we have

(8)(i) $\bar{\omega} \varsubsetneqq \omega_{N, \nu, j}^{*}$,

(ii) if $\omega_{N, \nu, j}^{*} \supsetneqq \omega_{N, \nu, \tilde{j}} \supset \bar{\omega},\left|a_{n\left(\omega_{N, \nu, j}^{*}\right)}\left(\omega_{N, \nu, \tilde{j}}\right)\right|<p^{-k+1} \lambda$, and

(iii) if $\bar{\omega}=\omega_{N, \nu, j}$ with $j<N, A_{n\left(\omega_{N, \nu, j}^{*}\right)}(\bar{\omega}) \geqslant p^{-k+1} \lambda$.

Let $\left(n\left(\omega_{N, \nu, j}^{*}\right), \omega_{N, \nu, j}^{*}\right) \in \Lambda_{k}^{*}(N, \nu)$ and

$$
\omega_{N, \nu, j+r} \in \prod_{N, \nu}\left(n\left(\omega_{N, \nu, j}^{*}\right), \omega_{N, \nu, j}^{*}, k\right) .
$$

For any $x \in \omega_{N, \nu, j+r^{\prime}}$ with $\omega_{N, \nu, j+r} \subset \omega_{N, \nu, j+r^{\prime}} \subset \omega_{N, \nu, j}^{*}$, we have the follow- 


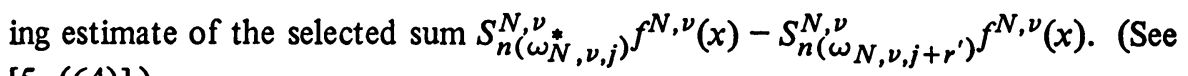
$[5,(64)]$.

For $\left(n\left(\omega_{N, \nu, j}^{*}\right), \omega_{N, \nu, j}^{*}\right) \in \Lambda_{k}^{*}(N, \nu)$, define

$$
\begin{aligned}
h^{N, \nu}(t) & =h^{N, \nu}\left(t, \Pi_{N, \nu}\left(n\left(\omega_{N, \nu, j}^{*}\right), \omega_{N, \nu, j}^{*}, k\right)\right) \\
& = \begin{cases}a_{n\left(\omega_{N, \nu, j}^{*}\right)}(\bar{\omega}) & \text { if } t \in \bar{\omega} \in \Pi_{N, \nu}, \\
0 & \text { if } t \notin \omega_{N, \nu, j}^{*} .\end{cases}
\end{aligned}
$$

Let the operator $W_{n}^{N, \nu}$ be defined by $W_{n}^{N, \nu} g=\overline{\chi_{n}^{N, \nu}} S_{n}^{N, \nu}\left(g \chi_{n}^{N, \nu}\right)$ for $g \in L^{1}(G)$. It follows that

$$
\begin{aligned}
& \left|S_{n\left(\omega_{N, \nu, j}^{N, \nu}\right)} f^{N, \nu}(x)-S_{n\left(\omega_{\left.N, \nu, j+r^{\prime}\right)}^{N, \nu}\right.} f^{N, \nu}(x)\right|
\end{aligned}
$$

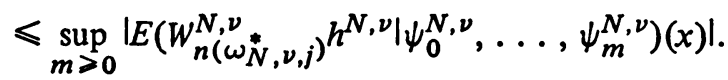

For convenience, the right-hand side of the above inequality will be denoted by

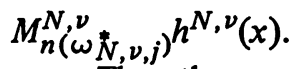

The other part of the exceptional set $E_{N, \nu}$ is used to control these selected sums. For $\left(n\left(\omega_{N, \nu, j}^{*}\right), \omega_{N, \nu, j}^{*}\right) \in \Lambda_{k}^{*}(N, v)$, define

$$
\begin{aligned}
& U_{N, \nu}\left(n\left(\omega_{N, \nu, j}^{*}\right), \omega_{N, \nu, j}^{*}, k\right) \\
& \quad=\left\{x \in \omega_{N, \nu, j}^{*}: M_{\left.n\left(\omega_{N, \nu, j}^{N, \nu}\right)^{*} h^{N, \nu}(x)>C_{0} L k p^{-k+1} \lambda\right\},}\right.
\end{aligned}
$$

where $C_{0}$ is an absolute constant to be determined later. Hence for $x \in \omega_{N, \nu, j+r^{\prime}}$ with $\omega_{N, \nu, j+r} \subset \omega_{N, \nu, j+r^{\prime}} \subset \omega_{N, \nu, j}^{*}, \omega_{N, \nu, j+r} \in \Pi_{N, \nu}\left(n\left(\omega_{N, \nu, j}^{*}\right), \omega_{N, \nu, j}^{*}, k\right)$, and $x \notin U_{N, \nu}\left(n\left(\omega_{N, \nu, j}^{*}\right), \omega_{N, \nu, j}^{*}, k\right)$, we have

$$
\left|S_{n\left(\omega_{N, \nu, j}^{N}\right)}^{N, \nu} f^{N, \nu}(x)-S_{n\left(\omega_{N, \nu, j+r^{\prime}}^{N, \nu}\right.}^{N} f^{N, \nu}(x)\right| \leqslant C_{0} L k p^{-k+1} \lambda .
$$

Let $E_{N, \nu}=V_{N, \nu} \cup \bigcup_{k=1}^{\infty} \bigcup_{\Lambda}^{*}(N, \nu) U_{N, \nu}\left(n\left(\omega_{N, \nu, j}^{*}\right), \omega_{N, \nu, j}^{*}, k\right)$. We will show that $x \notin E_{N, \nu}$ implies

$$
\left|S_{n}^{N, v} f^{N, \nu}(x)\right| \leqslant C L \lambda
$$

for $\nu m_{N} \leqslant n<(\nu+1) m_{N}$.

Before showing this we must make some technical adjustments. We first note that if $\nu m_{N} \leqslant n<(\nu+1) m_{N}$ and $p^{-k} \lambda \leqslant A_{n\left(\omega_{N, \nu, 0}\right)}\left(\omega_{N, \nu, 0}\right)<p^{-k+1} \lambda$, then $\left(n\left(\omega_{N, \nu, 0}\right), \omega_{N, \nu, 0}\right) \in \Lambda_{k}^{*}(N, \nu)$. Now suppose $\nu m_{N} \leqslant n<(\nu+1) m_{N}$, 
$p^{-k} \lambda \leqslant A_{n\left(\omega_{N, \nu, j}^{*}\right)}\left(\omega_{N, \nu, j}^{*}\right), 1 \leqslant j<N+1$, and $\omega_{N, \nu, j}^{*} \not \subset V_{N, \nu}$. Then there exists a triple $\left(\bar{n}, \omega_{N, \nu, \bar{j}}, \bar{k}\right)$ such that

(11)(i) $\bar{n}\left(\omega_{N, \nu, j}\right)=n\left(\omega_{N, \nu, j}\right)$

(ii) $\omega_{N, v, \bar{j}} \supset \omega_{N, v, j}$,

(iii) $1 \leqslant \bar{k} \leqslant k$, and

(iv) $\left(n\left(\omega_{N, \nu, \bar{j}}^{*}\right), \omega_{N, \nu, \bar{j}}^{*}\right) \in \Lambda_{\bar{k}}^{*}(N, v)$.

This is a consequence of (6) and (7). (See [5, (69)].)

If we choose $\left(\bar{n}, \omega_{N, \nu, j}, \bar{k}\right)$ satisfying (11) with $\bar{k}$ minimal, we have

$$
A_{\pi\left(\omega_{N, \nu, \tilde{j})}\right.}\left(\omega_{N, \nu, \tilde{j}}\right)<p^{-\bar{k}+1} \lambda
$$

for all $\omega_{N, \nu, \tilde{j}}$ such that $\omega_{N, \nu, j}^{*} \subset \omega_{N, \nu, \tilde{j}} \subset \omega_{N, \nu, \bar{j}}$. (See [5, (73)].)

We will now prove (10). We may assume $A_{n\left(\omega_{N, \nu, 0}\right)}\left(\omega_{N, v, 0}\right)>0$. Then there exists $\widetilde{k}_{0} \geqslant 1$ with $p^{-\widetilde{k}_{0}} \lambda \leqslant A_{n\left(\omega_{N, \nu, 0}\right)}<p^{-\widetilde{k}_{0}+1} \lambda$. Then $\left(n\left(\omega_{N, \nu, 0}\right), \omega_{N, \nu, 0}\right) \in \Lambda_{\widetilde{k}_{0}}^{*}(N, \nu)$ and the partition $\Pi_{N, \nu}\left(n\left(\omega_{N, \nu, 0}\right), \omega_{N, \nu, 0}, \widetilde{k}_{0}\right)$ is defined. Let $\omega_{N, \nu, j_{1}}^{*}$ be the element of this partition which contains $x$. Then (9) implies

$$
\left|S_{n}^{N, \nu} f^{N, \nu}(x)-S_{n\left(\omega_{N, \nu, j_{1}}^{N, \nu}\right)} f^{N, \nu}(x)\right| \leqslant C_{0} L \widetilde{k}_{0} p^{-\widetilde{k}_{0}+1} \lambda .
$$

If $j_{1}=N+1, n\left(\omega_{N, \nu, j_{1}}^{*}\right)=\nu m_{N}, S_{\nu m_{N}}^{N, \nu} f^{N, \nu}=0$ and we stop. If $j_{1}<N+1$, we have $A_{n\left(\omega_{N}^{*}, \nu, j_{1}\right)}\left(\omega_{N, \nu, j_{1}}^{*}\right) \geqslant p^{-\widetilde{k}_{0} 0^{+1}} \lambda$, by $(8)$. Choose $\left(n, \omega_{N, \nu, \bar{j}_{1}}, \widetilde{k}_{1}\right)$ as in (11) with $\widetilde{k}_{1}$ minimal. Then $\Pi_{N, \nu}\left(\bar{n}\left(\omega_{N, \nu, \bar{j}_{1}}^{*}\right), \omega_{N, \nu, \bar{j}_{1}}^{*}, \widetilde{k}_{1}\right)$ is defined. Let $\omega_{N, \nu, j_{2}}^{*}$ denote the element of this partition which contains $x$. We have $\omega_{N, \nu, j_{2}}^{*} \varsubsetneqq \omega_{N, \nu, j_{1}}^{*}, \bar{n}\left(\omega_{N, \nu, j_{2}}^{*}\right)=n\left(\omega_{N, \nu, j_{2}}^{*}\right)$, and, from (12) and (9)

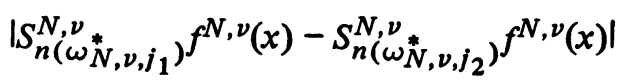

$$
\begin{aligned}
& \leqslant\left|S_{n\left(\omega_{N, \nu, j_{1}}^{N}\right)}^{N, \nu} f^{N, \nu}(x)-S_{n\left(\omega_{N, \nu, j_{1}}\right)}^{N, \nu} f^{N, \nu}(x)\right|
\end{aligned}
$$

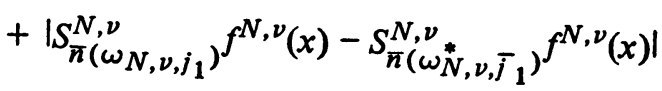

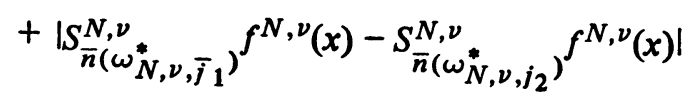

$$
\begin{aligned}
& \leqslant p^{-\tilde{k}_{1}+3} \lambda+2 C_{0} L \tilde{k}_{1} p^{-\tilde{k}_{1}+1} \lambda .
\end{aligned}
$$

(See $[5,(96)]$.$) If n\left(\omega_{N, \nu, j_{2}}^{*}\right)=\nu m_{N}$, we stop. Otherwise, we repeat the above step until we obtain 


$$
G=\omega_{N, \nu, 0}^{*} \supsetneqq \omega_{N, \nu, j_{1}}^{*} \supsetneq \ldots \supsetneqq \omega_{N, \nu, j_{\rho}}^{*}, \quad \tilde{k}_{0}>\tilde{k}_{1}>\ldots>\tilde{k}_{\rho} \geqslant 1 \text {, }
$$

with $n\left(\omega_{N, \nu, j_{i}}^{*}\right)>\nu m_{N}, i<\rho, n\left(\omega_{N, \nu, \rho}^{*}\right)=\nu m_{N}$, and

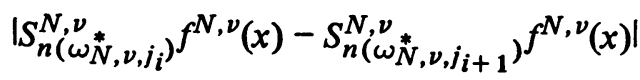

$$
\begin{aligned}
& \leqslant p^{-\tilde{k}_{i}+3} \lambda+2 C_{0} L \tilde{k}_{i} p^{-\tilde{k}_{i}+1} \lambda .
\end{aligned}
$$

Then

$$
\begin{aligned}
\left|S_{n}^{N, \nu} f^{N, \nu}(x)\right| & \leqslant \sum_{i=0}^{\rho-1} \mid S_{n\left(\omega_{N, \nu, j_{i}}^{N, \nu}\right)}^{* N, \nu}(x)-S_{n\left(\omega_{N, \nu, j_{i+1}}^{N, \nu} f^{N, \nu}(x) \mid\right.} \\
& \leqslant\left\{\left(\sum_{k=1}^{\infty} p^{-k+3}\right)+2 C_{0} L\left(\sum_{k=1}^{\infty} k p^{-k+1}\right)\right\} \lambda \\
& \leqslant C L \lambda .
\end{aligned}
$$

This completes the proof of (10).

Let $E=\bigcup_{N=1}^{\infty} \bigcup_{\nu=1}^{p_{N}^{-1}} E_{N, \nu}$. It follows from (10) that $x \notin E$ implies

$$
\left|S_{n}^{N, \nu} f^{N, \nu}(x)\right| \leqslant C L \lambda
$$

for $\nu m_{N} \leqslant n<(\nu+1) m_{N}, 1 \leqslant \nu<p_{N}$, and $N=1,2, \ldots$ The basic lemma will be proved if we show $\mu(E) \leqslant C_{q}^{q} \lambda^{-q} \int_{G}|f| q d \mu$, with $C_{q} \leqslant C q /(q-1)$.

We use Lemma 1 to estimate $\bigcup_{N=1}^{\infty} \bigcup_{\nu=1}^{p_{N-1}^{-1}} V_{N, \nu}$. Let $\omega_{N, \nu, j}^{*} \subset$ $\bigcup_{N=1}^{\infty} \bigcup_{\nu=1}^{p} N_{N, \nu}^{-1} ;$ then

$$
\begin{aligned}
& \lambda^{(q+1) / 2} \leqslant \mu\left(\omega_{N, \nu, j}\right)^{-1} \int_{\omega_{N, \nu, j}}|f|^{(q+1) / 2} d \mu \\
& \leqslant p \mu\left(\omega_{N, \nu, j}^{*}\right)^{-1} \int_{\omega_{N, \nu, j}^{*}}|f|(q+1) / 2 d \mu .
\end{aligned}
$$

Therefore

$$
\bigcup_{N=1}^{\infty} \bigcup_{\nu=1}^{p_{N-1}^{-1}} V_{N, \nu} \subset\left\{x \in G:\left(\mid f^{(q+1) / 2}\right)^{*}(x)>p^{-1} \lambda^{(q+1) / 2}\right\} .
$$

Applying Lemma 1 with $r=2 q /(q+1)$, we obtain

$$
\mu\left(\bigcup_{N=1}^{\infty} \bigcup_{\nu=1}^{p_{N-1}^{-1}} V_{N, \nu}\right) \leqslant C_{q}^{q} \lambda^{-q} \int_{G}|f|^{q} d \mu
$$

where $C_{q} \leqslant 2 p q /(q-1)$.

Next we follow the Carleson-Hunt method to estimate 


$$
\bigcup_{N=1}^{\infty} \bigcup_{\nu=1}^{p_{N}-1} \bigcup_{k=1}^{\infty} \bigcup_{\Lambda_{k}^{*}(N, \nu)} U_{N, \nu}\left(n\left(\omega_{N, \nu, j}^{*}\right), \omega_{N, \nu, h}^{*}, k\right) .
$$

It follows from (8) that $\left\|h^{N, \nu}\right\|_{\infty}<p^{-k+1} \lambda$. Estimates of the operators $M_{n\left(\omega_{N}^{*}, \nu, j\right)}^{N}$ then give

$$
\mu\left(U_{N, \nu}\left(n\left(\omega_{N, \nu, j}^{*}\right), \omega_{N, \nu, j}^{*}, k\right)\right) \leqslant C \exp \left(-C C_{0} L k\right) \mu\left(\omega_{N, \nu, j}^{*}\right) .
$$

(See $[5,(91)]$.) We choose $C_{0}$ so that

$$
\mu\left(U_{N, \nu}\left(n\left(\omega_{N, \nu, j}^{*}\right), \omega_{N, \nu, j}^{*}, k\right)\right) \leqslant p^{-5 L k} \mu\left(\omega_{N, \nu, j}^{*}\right) .
$$

We will also need an estimate of $\Sigma_{\Lambda *(N, v)} \mu\left(\omega_{N, \nu, j}\right)$. From the definition of $\Lambda_{k}(N, v)$ and Plancherel's formula, we have

$$
\sum_{\Lambda_{k}(N, \nu)} \mu\left(\omega_{N, \nu, j}\right) \leqslant p^{2 k} \lambda^{-2} \int_{G} \mid f^{N,\left.\nu\right|^{2}} d \mu .
$$

(See [5, (48)].) If $\Lambda_{k}^{*}(N, v)$ is nonempty, then there exists $\left(n\left(\omega_{N, \nu, j}\right), \omega_{N, \nu, j}\right) \in$ $\Lambda_{k}(n, \nu)$ with $\omega_{N, \nu, j}^{*} \notin V_{N, \nu}$. If $x \in \omega_{N, \nu, j}$, then (7) and an argument similar to that used in the proof of (6) give

$$
p^{-k} \lambda \leqslant \mu\left(\omega_{N, \nu, j}\right)^{-1} \int_{\omega_{N, \nu, j}}|f| d \mu .
$$

For $1<q<2$, it is a consequence of the fact that $f$ is a special function and the definition of $V_{N, \nu}$ that

$$
\begin{aligned}
p^{-k} \lambda & \leqslant \mu\left(\omega_{N, \nu, j}\right)^{-1} \int_{\omega_{N, \nu, j}}|f| d \mu \\
& \leqslant p^{(q+1) / 2} \mu\left(\omega_{N, \nu, j}\right)^{-1} \int_{\omega_{N, \nu, j}}|f|^{(q+1) / 2} d \mu \\
& \leqslant(p \lambda)^{(q+1) / 2} .
\end{aligned}
$$

Thus

$$
\lambda^{q-2} \leqslant p^{L k}, \quad k=1,2, \ldots,
$$

where $L=L(q)=\left[4 q^{2} /(q-1)\right]+1$. Therefore (15) implies

$$
\sum_{\Lambda_{k}^{*}(N, \nu)} \mu\left(\omega_{N, \nu, j}^{*}\right) \leqslant p^{5 k L-k} \lambda^{-q} \int_{G} \mid f^{N,\left.\nu\right|^{2}} d \mu .
$$

From (14) and (16), we have 


$$
\begin{gathered}
\mu\left(\bigcup_{N=1}^{\infty} \bigcup_{\nu=1}^{p_{N}^{-1}} \bigcup_{k=1}^{\infty} \bigcup_{\Lambda_{k}^{*}(N, \nu)} U_{N, \nu}\left(n\left(\omega_{N, \nu, j}^{*}\right), \omega_{N, \nu, j}^{*}, k\right)\right) \\
\leqslant \sum_{N=1}^{\infty} \sum_{\nu=1}^{p_{N}-1} \sum_{k=1}^{\infty} \sum_{\Lambda_{k}^{*}(N, \nu)} p^{-5 k L} \mu\left(\omega_{N, \nu, j}^{*}\right) \\
\leqslant\left(\sum_{k=1}^{\infty} p^{-k}\right) \lambda^{-q} \sum_{N=1}^{\infty} \sum_{\nu=1}^{p_{N}^{-1}} \int_{G} \mid f^{N, \nu \mid 2} d \mu .
\end{gathered}
$$

Using Plancherel's formula and the fact that $f$ is a special function, we obtain

$$
\left.\sum_{N=1}^{\infty} \sum_{\nu=1}^{N^{-1}} \int_{G}\left|f^{N,\left.\nu\right|^{2}} d \mu \leqslant \int_{G}\right| f\right|^{2} d \mu \leqslant \int_{G}|f|^{q} d \mu \text {. }
$$

Thus

$$
\mu\left(\bigcup_{N=1}^{\infty} \bigcup_{\nu=1}^{p_{N-1}^{-1}} \bigcup_{k=1}^{\infty} \bigcup_{\Lambda_{k}^{*}(N, \nu)} U_{N, \nu}\left(n\left(\omega_{N, \nu, j}^{*}\right), \omega_{N, \nu, j}^{*}, k\right)\right) \leqslant C^{q} \lambda^{-q} \int_{0}^{1}|f|^{q} d \mu
$$

(13) together with (17) give us the required estimate of $\mu(E)$. This completes proof of the basic lemma for the case $1<q<2$.

7. Proof of the basic lemma for the case $2 \leqslant q<\infty$. The proof is essentially the same as that in $\S 6$. The only differences lie in the definition of $V_{N, \nu}$ and the method of estimating $\Sigma_{\Lambda}^{*(N, \nu)}, \mu\left(\omega_{N, \nu, j}^{*}\right)$.

Define

$$
V_{N, \nu}=\bigcup_{j=1}^{N+1}\left\{\omega_{N, \nu, j}^{*}: \mu\left(\omega_{N, \nu, j}\right)^{-1} \int_{\omega_{N, \nu, j}} \mid f^{N, \nu \mid(q+1) / 2} d \mu \geqslant \lambda^{(q+1) / 2}\right\}
$$

We still have (6).

The construction of the rest of the exceptional set $E$ is exactly the same as that in the previous section. We also have $\left|S_{n}^{N, v} f^{N, v}(x)\right| \leqslant C L \lambda$, for $\nu m_{N} \leqslant$ $n<(\nu+1) m_{N}, 1 \leqslant \nu<p_{N}$ and $N=1,2, \ldots \ldots \cup_{N=1}^{\infty} \bigcup_{\nu=1}^{p_{N}^{-1}} V_{N, \nu}$ is estimated by Lemma 3. We have

$$
m\left(\bigcup_{N=1}^{\infty} \bigcup_{\nu=1}^{p_{N}^{-1}} V_{N, \nu}\right) \leqslant c^{q} \lambda^{-q} \int_{G}|f|^{q} d \mu
$$

We will now estimate $\bigcup_{\Lambda_{k}^{*}(N, \nu)} \mu\left(\omega_{N, \nu, j}^{*}\right)$. If $\left(n\left(\omega_{N, \nu, j}\right), \omega_{N, \nu, j}\right) \in$ $\Lambda_{k}(N, v)$, we have

$$
p^{-k} \lambda \leqslant \mu\left(\omega_{N, \nu, j}\right)^{-1} \int_{\omega_{N, \nu, j}}|f| d \mu \leqslant 1
$$


since $f$ is a special function. Hence $\lambda^{q-2} \leqslant p^{k L}, k=1,2, \ldots$ Therefore (15) implies (16).

The remainder of the proof follows as in the previous case. This completes the proof of the basic lemma for the case $2 \leqslant q<\infty$.

\section{REFERENCES}

1. L. A. Balašov, Series with respect to the Walsh system with monotone coefficients, Sibirsk. Mat. Ž. 12 (1971), 25-39 = Siberian Math. J. 12 (1971), 18-28. MR 44 \#1982.

2. A. P. Calderón and A. Zygmund, $A$ note on the interpolation of sublinear operations, Amer. J. Math. 78 (1956), 282-288. MR 18, 586.

3. L. Carleson, On convergence and growth of partial sums of Fourier series, Acta. Math. 116 (1966), 135-157. MR 33 \#7774.

4. K. L. Chung, A course in probability theory, Harcourt, Brace \& World, New York, 1968. MR 37 \#4842.

5. J. A. Gosselin, A. e. convergence of Vilenkin-Fourier series, Trans. Amer. Math. Soc. 185 (1973), 345-370.

6. R. A. Hunt, On the convergence of Fourier series, Orthogonal Expansions and their Continuous Analogues (Proc. Conf., Edwardsville, Ill., 1967), Southern Illinois Univ. Press, Carbondale, Ill., 1968, pp. 235-255. MR 38 \#6296.

7. - Almost everywhere convergence of Walsh-Fourier series of $L^{2}$ functions, Proc. Internat. Congress Math. (Nice, 1970), vol. 2, Gauthier-Villars, Paris, 1971, pp. 655-661.

8. R. A. Hunt and M. H. Taibleson, Almost everywhere convergence of Fourier series on the ring of integers of a local field, SIAM J. Math. Anal. 2 (1971), 607-625. MR 47 \#18.

9. K. H. Moon, Maximal functions related to certain linear operators, Doctoral Dissertation, Purdue University, West Lafayette, Ind., 1972.

10. P. Sjölin, An inequality of Paley and convergence a. e. of Walsh-Fourier series, Ark. Mat. 7 (1969), 551-570. MR 39 \#3222.

11. E. M. Stein, Topics in harmonic analysis related to the Littlewood-Paley theory, Ann. of Math. Studies, no. 63, Princeton Univ. Press, Princeton, N. J.; Univ. of Tokyo Press, Tokyo, 1970. MR $40 \# 6176$.

12. N. Ja. Vilenkin, On a class of complete orthonormal systems, Amer. Math. Soc. Transl. (2) 28 (1963), 1-35. MR 27 \#4001.

13. D. Waterman, W-systems are the Walsh functions, Bull. Amer. Math. Soc. 75 (1969), 139-142. MR 38 \#2514.

14. W. S. Young, Maximal inequalities and almost everywhere convergence, Doctoral Dissertation, Purdue University, West Lafayette, Ind., 1973.

15. W.-S. Young, On rearrangements of Walsh-Fourier series and Hardy-Littlewood type maximal inequalities, Bull. Amer. Math. Soc. 80 (1974), 490-494.

16. - On the a. e. convergence of Walsh-Kaczmarz-Fourier series, Proc. Amer. Math. Soc. 44 (1974), 353-358.

DEPARTMENT OF MATHEMATICS, SYRACUSE UNIVERSITY, SYRACUSE, NEW YORK 13210

DEPARTMENT OF MATHEMATICS, NORTHWESTERN UNIVERSITY, EVANSTON, ILLINOIS 60201

Current address (J. A. Gosselin): Department of Mathematics, University of Georgia, Athens, Georgia 30601

Current address (W. S. Young): Department of Mathematics, University of Chicago, Chicago, Illinois 60637 